\title{
Associations of Handgrip Strength and Handgrip Strength Asymmetry With Depression in the Elderly in Korea: A Cross-sectional Study
}

\author{
Kyungduk Hurh, Yoonsik Park, Gyu Ri Kim, Sung-In Jang, Eun-Cheol Park \\ Department of Preventive Medicine and Institute of Health Services Research, Yonsei University College of Medicine, Seoul, Korea
}

Objectives: Recent studies have suggested that assessing handgrip strength (HGS) asymmetry together with HGS may be helpful for evaluating problems in geriatric patients. This study aimed to identify whether HGS asymmetry, weakness, or both were associated with depression in Korean older adults.

Methods: This study included 4274 subjects from the sixth and seventh Korea National Health and Nutrition Examination Survey. Depression was measured using the Patient Health Questionnaire-9. The maximum HGS of the dominant hand was used as a representative value. HGS symmetry was categorized by the ratio of the HGS of the dominant hand to that of non-dominant hand. The odds ratio (OR) for depression was calculated according to the HGS and its symmetry.

Results: In total, 240 (12.5\%) men and 534 (22.7\%) women had depression. HGS or HGS asymmetry showed no statistically significant associations with depression in elderly men. Elevated odds of depression were observed in elderly women with low HGS (OR, 1.93; $95 \%$ confidence interval [CI], 1.33 to 2.81 ) or prominent $\mathrm{HGS}$ asymmetry (OR, 1.46; $95 \% \mathrm{Cl}, 1.02$ to 2.08). There was a positive additive interaction between asymmetric HGS and weakness, as women with low and prominently asymmetric HGS showed higher odds of depression (OR, 3.77; $95 \% \mathrm{Cl}, 2.16$ to 6.59$)$ than women with high and symmetric HGS.

Conclusions: Depression in elderly Korean women was associated with both low and asymmetric HGS. Our findings support the potential value of HGS asymmetry as an indicator of HGS.

Key words: Hand strength, Depression, Women, Aged, Mental health, Korea National Health and Nutrition Examination Survey

\section{INTRODUCTION}

Due to the rapid growth in the elderly population and a low birth rate, Korea became an aged society in 2018, with 7.37

Received: July 6, 2020 Accepted: December 14, 2020

Corresponding author: Eun-Cheol Park

Department of Preventive Medicine and Institute of Health Services

Research, Yonsei University College of Medicine, 50-1 Yonsei-ro,

Seodaemun-gu, Seoul 03722, Korea

E-mail: ecpark@yuhs.ac

This is an Open Access article distributed under the terms of the Creative Commons Attribution Non-Commercial License (https://creativecommons.org/licenses/bync/4.0// which permits unrestricted non-commercial use, distribution, and reproduction in any medium, provided the original work is properly cited. million people aged over 65 years, exceeding 14\% of the total population. By 2025, Korea will become a post-aged society, with the elderly accounting for over $20 \%$ of the total population [1].

Older adults have variety of health problems including mental problems. Depression is one of the most common mental problems and important social issues facing the elderly in Korea. According to previous studies, the overall prevalence of late-life depression in Korea exceeds $10 \%$ and is expected to increase [2]. Additionally, in 2017, the suicide rate among the elderly in Korea was 47.7 per 100000 people, nearly 2 times higher than that of young people [3] and 4.3 times higher than the Organization for Economic Cooperation and Devel- 
opment average [4].

Sarcopenia, which is defined as loss of muscle mass and strength with age, is becoming recognized as a major cause of disability and morbidity in the elderly population [5]. Measuring handgrip strength (HGS) is considered to be an effective and convenient way to measure muscle strength. Owing to its cost-effectiveness, accessibility, and ease of use, measuring HGS is the most widely used method to evaluate muscle strength in Asian sarcopenia research [6]. Previous studies have reported that low HGS was associated with incident disability, metabolic syndrome, cardiovascular mortality, and allcause mortality in the elderly [7-9]. Moreover, low HGS has also been reported to be associated with mental health problems such as cognitive impairment and depression [10-13].

Normally, there is a slight difference in grip strength between both hands, typically ranging from $0 \%$ to $30 \%$ [14]. Studies have suggested that prominent unilateral HGS may be related to mental disorders such as schizophrenia, bipolar disorder, and depression [15-17]. However, Yu et al. [18] suggested that the degree, not direction, of HGS asymmetry was related to depression in the elderly. Moreover, several studies have found that HGS asymmetry and weakness were jointly associated with functional impairment or lower cognitive function in the elderly [19-21]. To the best of our knowledge, no report has considered the effect of low HGS together with HGS asymmetry on depression in the Korean population. Therefore, this study aimed to determine whether low HGS, asymmetric HGS, or both are associated with depression in this population.

\section{METHODS}

\section{Study Sample}

The study data were collected from the 2014, 2016, and 2018 Korea National Health and Nutrition Examination Survey (KNHANES VI and VII). The KNHANES is a cross-sectional, nationally representative survey conducted by the Korea Centers for Disease Control and Prevention (KCDC).

Details on the sampling design of the KNHANES are available on the KNHANES webpage (https://knhanes.cdc.go.kr/knhanes/ eng/index.do). The KNHANES targets non-institutionalized Korean citizens living in Korea. A multi-stage clustered probability design was used to sample the survey participants. For example, in the seventh KNHANES survey, 192 primary sampling units (PSUs) were extracted from roughly 200000 geographi-
2014, 2016, 2018 Korea National Health and

Nutrition Examination Survey ( $n=23$ 692)

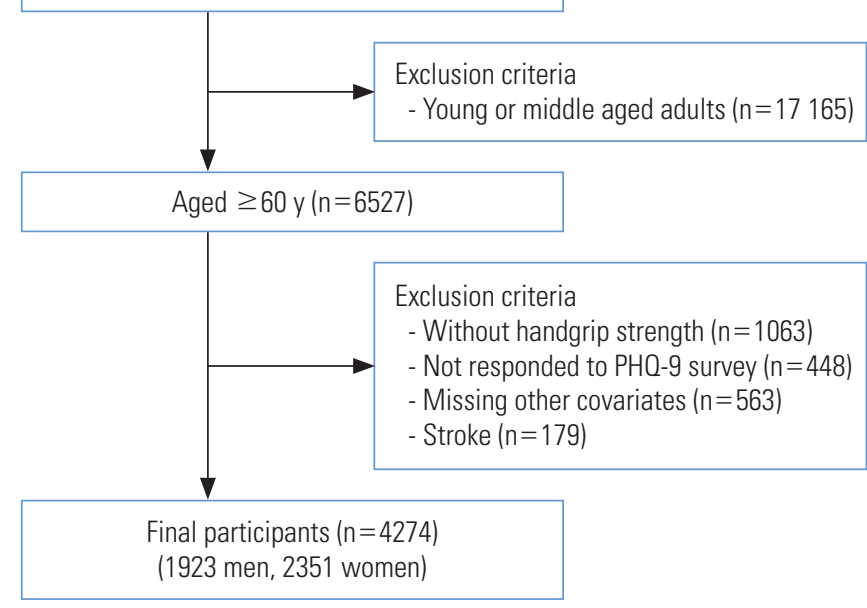

Figure 1. Flow chart of participant selection. PHQ-9, Patient Health Questionnaire-9.

cally determined PSUs for the entire country. Using systematic sampling, 23 final target households were drawn from each PSU, which comprised approximately 60 households. Finally, individuals aged 1 year and over were surveyed in the selected households [22]. Approximately 10000 individuals are targeted for the KNHANES each year, with a response rate of roughly $75 \%$.

A total of 23692 individuals responded and were surveyed for the KNHANES in 2014, 2016, and 2018. The 2015 and 2017 data were excluded as they did not contain a depression screening survey. Of those who responded, participants who were under the age of 60 years or had any incomplete data or missing values were excluded. Participants with stroke were also excluded because this condition may cause muscle weakness and act as a confounder. In the final sample, the total number of participants was 4274 (Figure 1).

\section{Depression}

To select participants with depression, we used the Patient Health Questionnaire-9 (PHQ-9). The PHQ-9 is a diagnostic instrument for depression, which scores each of the 9 Diagnostic and Statistical Manual of Mental Disorders (DSM)-IV criteria from 0 (not at all) to 3 (nearly every day) [23]. Since the core criteria for major depressive disorder have not changed in the DSM-V, the effectiveness of the PHQ-9 was maintained. In this study, we used a PHQ-9 score of 5 as a threshold for the diagnosis of depression, which was adjusted for the Korean elderly [24]. 


\section{Handgrip Strength}

HGS was measured 3 times for each hand by a skilled investigator, using a digital dynamometer. The participants were instructed to grip the instrument with their maximum strength for 3 seconds in the upright position, and a 60-second break was allowed after each cycle. The maximum HGS of the dominant hand was used as a representative value. We categorized HGS into 3 levels: high (60-100th percentile of the study population), medium (20-60th percentile of the study population), and low (lower than 20th percentile of the study population). The cut-off value of low HGS followed the criteria recommended by the Asian Working Group of Sarcopenia [6]. It has been assumed that the grip strength of the dominant hand is approximately $10 \%$ greater than that of the non-dominant hand, which is referred to as the "10\% rule" [19-21,25]. Therefore, symmetric HGS was defined as a calculated HGS ratio between 0.9 and 1.1; otherwise, HGS was defined as asymmetric. We classified asymmetric HGS as moderate (ratio of $0.8-0.9$ or 1.11.2) or prominent (ratio lower than 0.8 or higher than 1.2) to investigate whether the degree of HGS asymmetry was related to the prevalence of depression.

\section{Covariates}

All data were stratified by gender. Age $(60-64,65-69,70-74$, 75 or older), area of residence (metropolitan, rural), educational level (high school or below, college or above), job, household income (quartile of household income from the total 2014, 2016, and 2018 KNHANES survey participants), and marital status (with spouse, without spouse) were included as demographic and socioeconomic factors. The participants' jobs were classified into 4 categories: white-collar (professional, administrative, or office-work jobs), pink-collar (jobs in the service industry), blue-collar (unskilled manual labor, farming or fishing, machine operating, or other physical work), or none (unemployed, housewives, or students) [26]. Eating habits (regular, irregular), lifetime smoking experience (yes, no), frequency of drinking (2-4 times/wk, 2-4 times/mo, never or occasionally), obesity (body mass index $\geq 25 \mathrm{~kg} / \mathrm{m}^{2},<25 \mathrm{~kg} / \mathrm{m}^{2}$ ), physical activity (sufficient, insufficient), degree of feeling stressed (a lot, a little), and presence of comorbid chronic diseases were included as health-related factors. Sufficient or insufficient physical activity was defined by the Global Physical Activity Questionnaire, developed by the World Health Organization [27]. Participants were classified as having a chronic disease if they had hypertension, type 2 diabetes mellitus, or dyslipidemia.

\section{Statistical Analysis}

The general characteristics of the participants are presented as number and percentage according to depression. The chisquare test was performed to determine the significance of differences in baseline characteristics between participants with depression and those without depression. We investigated the association between HGS-related factors (HGS, HGS symmetry, and their joint action) and depression in the elderly using a multiple logistic regression model $\left(R^{2}=0.13\right.$, max-rescaled $R^{2}=0.22$ ). The variance inflation factor $(V I F)$ was calculated to investigate the collinearity of independent variables. We determined that all calculated VIF values were less than 3.0, indicating acceptable multicollinearity among the independent variables. The approximate relative excess risk due to interaction from the odds ratio (RERloR) was calculated to evaluate the synergistic effect of HGS asymmetry and weakness [28]. Two exposures can be interpreted as having a positive additive interaction when the $\mathrm{RER}_{\text {oR }}$ is larger than 0 , while a negative additive interaction is shown if the RERI than 0 [28]. Subgroup analyses stratified by each independent variable were performed to examine the independent effects of socioeconomic and health-related factors. Lastly, because the amount of missing data was quite large and the variables seemed not to be missing completely at random, we conducted additional analyses using the not missing completely at random (NOMCAR) option in SAS $[29,30]$. All statistical analyses were performed using SAS version 9.4 (SAS Institute Inc., Cary, NC, USA).

\section{Ethics Statement}

The KNHANES has been reviewed annually and approved by the KCDC Research Ethics Review Committee since 2007 and the collected data are published online for researchers to use. Before participation, written informed consent was obtained from all survey respondents. The committee operates according to the KCDC Research Ethics Review Committee's standard guidelines. Since this study used publicly available data, institutional review board approval was not required for this study based on Article 2.2 of the Enforcement Rule of the Bioethics and Safety Act in Korea.

\section{RESULTS}

Table 1 shows the general characteristics of participants according to the presence of depression. In total, 4274 partici- 
Table 1. General characteristics of the study subjects

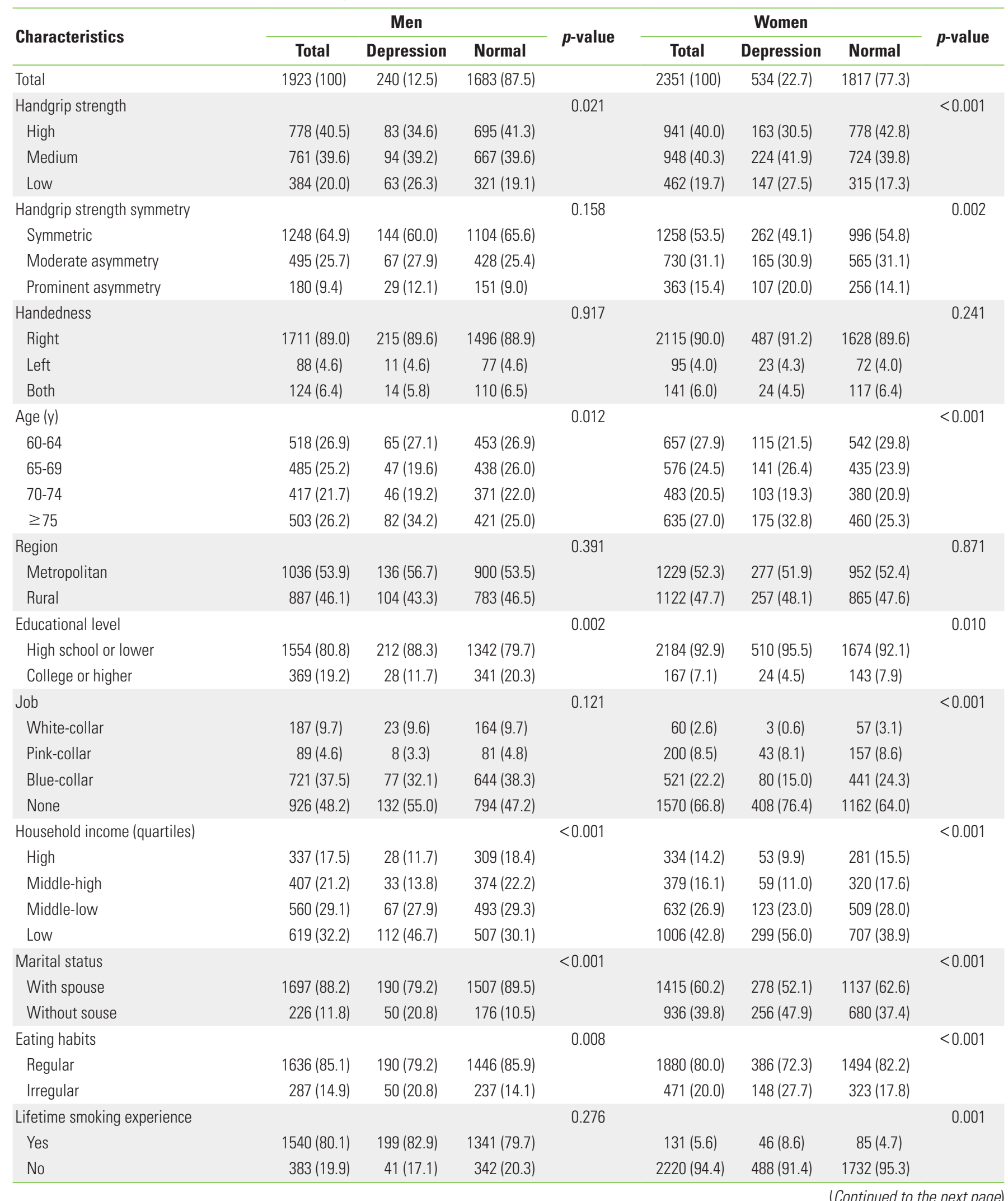


Table 1. Continued from the previous page

\begin{tabular}{|c|c|c|c|c|c|c|c|c|}
\hline \multirow{2}{*}{ Characteristics } & \multicolumn{3}{|c|}{ Men } & \multirow{2}{*}{$p$-value } & \multicolumn{3}{|c|}{ Women } & \multirow{2}{*}{$p$-value } \\
\hline & Total & Depression & Normal & & Total & Depression & Normal & \\
\hline Drinking & & & & 0.662 & & & & 0.614 \\
\hline 2-4 times/wk & $675(35.1)$ & $83(34.6)$ & $592(35.2)$ & & $137(5.8)$ & $35(6.6)$ & $102(5.6)$ & \\
\hline 2-4 times/mo & $388(20.2)$ & $44(18.3)$ & $344(20.4)$ & & $182(7.7)$ & $38(7.1)$ & $144(7.9)$ & \\
\hline Never or occasionally & $860(44.7)$ & $113(47.1)$ & $747(44.4)$ & & $2032(86.4)$ & $461(86.3)$ & $1571(86.5)$ & \\
\hline $\mathrm{BMI}\left(\mathrm{kg} / \mathrm{m}^{2}\right)^{1}$ & & & & 0.025 & & & & 0.290 \\
\hline Obese $(\geq 25)$ & 639 (33.2) & $64(26.7)$ & $575(34.2)$ & & $929(39.5)$ & $200(37.5)$ & $729(40.1)$ & \\
\hline Normal weight or underweight $(<25)$ & $1284(66.8)$ & $176(73.3)$ & $1108(65.8)$ & & $1422(60.5)$ & $334(62.5)$ & $1088(59.9)$ & \\
\hline Physical activity & & & & 0.068 & & & & 0.004 \\
\hline Sufficient & $822(42.7)$ & $89(37.1)$ & $733(43.6)$ & & $775(33.0)$ & $148(27.7)$ & $627(34.5)$ & \\
\hline Insufficient & 1101 (57.3) & 151 (62.9) & $950(56.4)$ & & $1576(67.0)$ & 386 (72.3) & $1190(65.5)$ & \\
\hline Stress & & & & $<0.001$ & & & & $<0.001$ \\
\hline A lot & $220(11.4)$ & $88(36.7)$ & $132(7.8)$ & & $484(20.6)$ & $232(43.4)$ & 252 (13.9) & \\
\hline A little & 1703 (88.6) & $152(63.3)$ & 1551 (92.2) & & 1867 (79.4) & $302(56.6)$ & 1565 (86.1) & \\
\hline Chronic disease $^{2}$ & & & & 0.073 & & & & 0.070 \\
\hline Yes & $1103(57.4)$ & $151(62.9)$ & $952(56.6)$ & & 1487 (63.2) & $356(66.7)$ & 1131 (62.2) & \\
\hline No & $820(42.6)$ & $89(37.1)$ & $731(43.4)$ & & 864 (36.8) & $178(33.3)$ & 686 (37.8) & \\
\hline
\end{tabular}

$\mathrm{BMI}$, body mass index.

'Obesity status was defined by BMI based on 2014 Clinical Practice Guidelines for Overweight and Obesity in Korea.

${ }^{2}$ Chronic diseases included hypertension, diabetes mellitus, and dyslipidemia.

pants were included in the study, of whom 1923 (45.0\%) were men and 2351 (55.0\%) were women. The number of participants classified as having depression based on a PHQ-9 score of 5 or higher was $240(12.5 \%)$ men and 534 (22.7\%) women.

For men, there was no statistically significant association between HGS or HGS symmetry and depression. In contrast, compared to women with high HGS, the odds ratio (OR) for depression in women with medium HGS was 1.51 (95\% confidence $[\mathrm{Cl}], 1.13$ to 2.00 ) and that of women with a low HGS was $1.93(95 \% \mathrm{Cl}, 1.33$ to 2.81$)$. The OR for depression in women with prominent HGS asymmetry was $1.46(95 \% \mathrm{Cl}, 1.02$ to 2.08), compared to women with symmetric HGS. Other covariates were associated with depression among women, such as low household income, living without a spouse, irregular eating habits, and experiencing a high level of stress (Table 2).

Table 3 shows the joint action of HGS and HGS symmetry. No statistically significant association was found for men. Compared to women with high and symmetric HGS, the OR for depression was $3.77(95 \% \mathrm{Cl}, 2.16$ to 6.59$)$ in women with prominently asymmetric and low $\mathrm{HGS}$ and $2.02(95 \% \mathrm{Cl}, 1.14$ to 3.58$)$ and $1.96(95 \% \mathrm{Cl}, 1.19$ to 3.23$)$ in women with moderately asymmetric and low HGS and prominently asymmetric and medium HGS, respectively. Although the association was not statistically significant, the OR for depression was $1.53(95 \% \mathrm{Cl}, 0.93$ to
Table 2. Handgrip strength and other factors associated with depression in the Korean elderly

\begin{tabular}{|c|c|c|}
\hline \multirow{2}{*}{ Variables } & \multicolumn{2}{|c|}{ Depression (PHO-9 $\geq 5$ ) } \\
\hline & Men & Women \\
\hline \multicolumn{3}{|l|}{ Handgrip strength } \\
\hline High & 1.00 (reference) & 1.00 (reference) \\
\hline Medium & $0.97(0.62,1.53)$ & $1.51(1.13,2.00)$ \\
\hline Low & $0.94(0.56,1.57)$ & $1.93(1.33,2.81)$ \\
\hline \multicolumn{3}{|c|}{ Handgrip strength asymmetry } \\
\hline Symmetric & 1.00 (reference) & 1.00 (reference) \\
\hline Moderate asymmetry & $1.28(0.87,1.88)$ & $1.14(0.86,1.52)$ \\
\hline Prominent asymmetry & $1.20(0.71,2.05)$ & $1.46(1.02,2.08)$ \\
\hline \multicolumn{3}{|l|}{ Handedness } \\
\hline Right & 1.00 (reference) & 1.00 (reference) \\
\hline Left & $1.03(0.49,2.16)$ & $1.08(0.60,1.94)$ \\
\hline Both & $0.66(0.28,1.55)$ & $0.66(0.37,1.18)$ \\
\hline \multicolumn{3}{|l|}{ Age (y) } \\
\hline $60-64$ & 1.00 (reference) & 1.00 (reference) \\
\hline $65-69$ & $0.59(0.33,1.04)$ & $1.19(0.81,1.74)$ \\
\hline $70-74$ & $0.75(0.42,1.33)$ & $0.67(0.44,1.00)$ \\
\hline$\geq 75$ & $1.08(0.59,1.97)$ & $0.80(0.52,1.22)$ \\
\hline \multicolumn{3}{|l|}{ Region } \\
\hline Metropolitan & 1.00 (reference) & 1.00 (reference) \\
\hline Rural & $0.86(0.60,1.24)$ & $0.83(0.64,1.07)$ \\
\hline
\end{tabular}

(Continued to the next page) 
Table 2. Continued from the previous page

\begin{tabular}{|c|c|c|}
\hline \multirow{2}{*}{ Variables } & \multicolumn{2}{|c|}{ Depression (PHQ-9 $\geq 5$ ) } \\
\hline & Men & Women \\
\hline \multicolumn{3}{|l|}{ Educational level } \\
\hline High school or lower & $1.91(1.09,3.35)$ & $1.48(0.89,2.44)$ \\
\hline College or higher & 1.00 (reference) & 1.00 (reference) \\
\hline \multicolumn{3}{|l|}{ Job } \\
\hline White-collar & 1.00 (reference) & 1.00 (reference) \\
\hline Pink-collar & $0.70(0.28,1.75)$ & $2.69(0.76,9.49)$ \\
\hline Blue-collar & $0.67(0.34,1.32)$ & $1.74(0.52,5.78)$ \\
\hline None & $0.83(0.42,1.63)$ & $3.07(0.94,10.03)$ \\
\hline \multicolumn{3}{|l|}{ Household income (quartiles) } \\
\hline High & 1.00 (reference) & 1.00 (reference) \\
\hline Middle-high & $1.25(0.67,2.31)$ & $0.94(0.60,1.49)$ \\
\hline Middle-low & $1.61(0.88,2.96)$ & $1.26(0.83,1.92)$ \\
\hline Low & $2.11(1.08,4.13)$ & $1.80(1.16,2.82)$ \\
\hline \multicolumn{3}{|l|}{ Marital status } \\
\hline With spouse & 1.00 (reference) & 1.00 (reference) \\
\hline Without souse & $1.79(1.13,2.84)$ & $1.34(1.01,1.78)$ \\
\hline \multicolumn{3}{|l|}{ Eating habits } \\
\hline Regular & 1.00 (reference) & 1.00 (reference) \\
\hline Irregular & $1.26(0.79,2.01)$ & $1.58(1.19,2.09)$ \\
\hline \multicolumn{3}{|l|}{ Lifetime smoking experience } \\
\hline Yes & $1.24(0.77,1.99)$ & $1.26(0.76,2.10)$ \\
\hline No & 1.00 (reference) & 1.00 (reference) \\
\hline \multicolumn{3}{|l|}{ Drinking } \\
\hline 2-4 times/wk & $0.86(0.57,1.30)$ & $1.24(0.74,2.08)$ \\
\hline 2-4 times/mo & $1.07(0.67,1.71)$ & $0.96(0.58,1.57)$ \\
\hline Never or occasionally & 1.00 (reference) & 1.00 (reference) \\
\hline \multicolumn{3}{|l|}{$\mathrm{BMI}\left(\mathrm{kg} / \mathrm{m}^{2}\right)^{1}$} \\
\hline Obese ( $\geq 25)$ & $0.75(0.51,1.09)$ & $0.80(0.62,1.04)$ \\
\hline Normal weight or underweight $(<25)$ & 1.00 (reference) & 1.00 (reference) \\
\hline \multicolumn{3}{|l|}{ Physical activity } \\
\hline Sufficient & 1.00 (reference) & 1.00 (reference) \\
\hline Insufficient & $1.06(0.75,1.51)$ & $1.22(0.91,1.64)$ \\
\hline \multicolumn{3}{|l|}{ Stress } \\
\hline A lot & $7.79(5.21,11.65)$ & $4.60(3.53,5.99)$ \\
\hline A little & 1.00 (reference) & 1.00 (reference) \\
\hline \multicolumn{3}{|l|}{ Chronic disease $^{2}$} \\
\hline Yes & $1.36(0.95,1.96)$ & $1.12(0.85,1.47)$ \\
\hline No & 1.00 (reference) & 1.00 (reference) \\
\hline
\end{tabular}

PHQ-9, Patient Health Questionnaire-9; BMI, body mass index.

'Obesity status was defined by BMI based on 2014 Clinical Practice Guidelines for Overweight and Obesity in Korea.

${ }^{2}$ Chronic diseases included hypertension, diabetes mellitus, and dyslipidemia.

2.52) in women with moderately asymmetric and medium HGS and $1.47(95 \% \mathrm{Cl}, 0.99$ to 2.20$)$ in women with symmetric and medium HGS. However, the odds of having depression were
Table 3. Joint action of HGS and HGS symmetry ${ }^{1}$ for depression

\begin{tabular}{lccc}
\hline \multirow{2}{*}{ Variables } & \multicolumn{3}{c}{ Depression (PHQ-9 $\geq \mathbf{5})$} \\
\cline { 2 - 4 } & Symmetric & $\begin{array}{c}\text { Moderate } \\
\text { asymmetry }\end{array}$ & $\begin{array}{c}\text { Prominent } \\
\text { asymmetry }\end{array}$ \\
\hline Men & $1.00($ reference) & $1.40(0.76,2.59)$ & $1.23(0.38,4.04)$ \\
High & $1.03(0.60,1.76)$ & $1.22(0.62,2.41)$ & $1.14(0.47,2.79)$ \\
Medium & $0.96(0.51,1.84)$ & $1.20(0.55,2.62)$ & $1.23(0.51,2.97)$ \\
Low & & & \\
Women & $1.00($ reference) & $1.17(0.73,1.88)$ & $0.83(0.45,1.55)$ \\
High & $1.47(0.99,2.20)$ & $1.53(0.93,2.52)$ & $1.96(1.19,3.23)$ \\
Medium & $1.45(0.82,2.56)$ & $2.02(1.14,3.58)$ & $3.77(2.16,6.59)$ \\
\hline Low &
\end{tabular}

Values are presented as odds ratio (95\% confidence interval).

HGS, handgrip strength; PHO-9, Patient Health Questionnaire-9; RERI $\mathrm{OR}_{\text {, rela- }}$ tive excess risk due to interaction from odds ratios.

'Calculated RERI oR: 1.86 for women with prominent HGS asymmetry and low HGS, 0.76 for women with moderate HGS asymmetry and low HGS, 0.60 for women with prominent HGS asymmetry and medium HGS, and 0.28 for women with moderate HGS asymmetry and medium HGS. All of the above RERlor values were statistically significant.

not higher in women with high HGS and HGS asymmetry than in women with high and symmetric HGS. The calculated RERI was $1.86(95 \% \mathrm{Cl}, 1.83$ to 1.89$)$ for women with prominent HGS asymmetry and low $\mathrm{HGS}, 0.76(95 \% \mathrm{Cl}, 0.74$ to 0.77$)$ for those with moderate HGS asymmetry and low HGS, $0.60(95 \% \mathrm{Cl}$, 0.59 to 0.61 ) for those with prominent HGS asymmetry and medium HGS, and $0.28(95 \% \mathrm{Cl}, 0.28$ to 0.28$)$ for those with moderate HGS asymmetry and medium HGS. Asymmetric HGS and low HGS had a positive additive interaction effect on the prevalence of depression, and that interaction effect became stronger with the degree of HGS asymmetry and weakness.

We analyzed the relationship between HGS and depression according to subgroups for each independent variable. Supplemental Material 1 shows the significant results. Compared to women with high HGS in the same groups, the odds of depression among women with low HGS were higher among those who were older than 75 years $(\mathrm{OR}, 2.89,95 \% \mathrm{Cl}, 1.46$ to $5.74)$, those who were normal-weight or underweight (OR, 2.18; $95 \% \mathrm{Cl}, 1.36$ to 3.50$)$, those who engaged in regular physical activity (OR, 2.24; $95 \% \mathrm{Cl}, 1.14$ to 4.38), and those who had a chronic illness (OR, $2.41 ; 95 \% \mathrm{Cl}, 1.52$ to 3.80$)$.

\section{DISCUSSION}

This study investigated the associations of HGS and HGS symmetry with depression among the elderly in Korea. We found that asymmetric HGS, as well as low HGS, showed a statistically 
significant relationship with the prevalence of depression among elderly Korean women after adjusting for socioeconomic and health-related factors.

Previous studies have explained the association between low HGS and depression through several mechanisms. Fukumori et al. [31] found a longitudinal relationship between HGS and depression in a Japanese cohort study among adults aged 4079. Low HGS represents low physical function, which can lead to poor quality of life and adversely affect mental health in the long term. On the contrary, depression can be a risk factor for developing sarcopenia because people with depression often engage in less physical activity and have a lower appetite than others. Both of the above proposals may be correct, and there may be a bidirectional relationship between low HGS and depression. Chronic illnesses are associated with both decreased muscle strength and depression and can act as a pathway between them $[8,9,32]$.

In the present study, statistically significant associations were observed only in women. This can be explained by the fact that women are known to be more susceptible to depression than men and thus are more sensitive to factors associated with depression. Since muscle strength differs by gender, an HGS of $28.1 \mathrm{~kg}$ was in the lower 20th percentile for men, while the same strength was over the 90th percentile for women in this study. In other words, men with low muscle strength are still stronger than women with normal muscle strength. Therefore, low muscle strength may have less impact on the physical function and quality of life of men than of women.

Previous studies found that the prevalence of depression was higher in left-handed people, corresponding to a functional brain abnormality (namely, hyperactivity of the right hemisphere) [33]. Crews Jr et al. [15] found that depressed women displayed significantly less hand-grip fatigue of the left hand than non-depressed women. However, in our results, there were no difference in the prevalence of depression between left-handed and right-handed participants (Table 2). In addition, among right-handed women, the odds of depression increased as the degree of asymmetry increased, regardless of its direction, compared to women with symmetric HGS (Supplemental Material 2). If the association between asymmetric HGS and depression is a result of hyperactivation of the right hemisphere, the asymmetry associated with depression would only appear as an increase in the left HGS. Moreover, compared to women with symmetric HGS, the ORs for depression in women with HGS asymmetry were almost the same in the unadjust- ed model, the model adjusted for socioeconomic factors, and the fully adjusted model (Supplemental Material 3). Therefore, these results suggest that HGS asymmetry is associated with depression in the elderly through a mechanism other than brain functional abnormality, regardless of socioeconomic and health-related lifestyle factors.

McGrath et al. [19] found that HGS asymmetry was associated with functional limitations in older Americans in their crosssectional study. Therefore, asymmetric HGS may represent an asymmetry in motor function, which leads to physical imbalance and functional limitations. Subsequently, a decline in the quality of life and depression can be induced by functional limitations.

As shown in Table 3, the differences in the ORs for having depression according to HGS symmetry increased with decreasing HGS. The calculated RERlor was $1.86(95 \% \mathrm{Cl}, 1.83$ to 1.89) for women with prominent HGS asymmetry and a low HGS and was larger than 0 in all other groups of women with asymmetric and lower HGS. If the RERIor is larger than 0 , it can be interpreted as showing that 2 exposures have a positive additive interaction. Thus, a positive additive interaction for depression was found to be present between low HGS and HGS asymmetry, and this interaction was particularly large between low HGS and prominently asymmetric HGS. However, the association between HGS asymmetry and depression appeared only in elderly women with lower HGS. Frailty is an emerging paradigm composed of characteristics including wasting (loss of muscle mass and strength, weight loss), loss of endurance, decreased balance and mobility, slowed performance, relative inactivity, and, potentially, diminished cognitive function [34]. Many studies have shown a reciprocal interaction between depression and frailty in older adults, each condition may be a risk factor for the development of the other $[35,36]$. It has been reported that asymmetry of lower leg strength is associated with frailty phenotypes including standing imbalance, decreased walking speed, and falls in the elderly $[37,38]$. Because HGS is closely related to lower limb strength $[39,40]$, we think that asymmetric and low HGS can be integrated into the common process of frailty, which may act as a linking pathway to depression and show synergistic interactions by sharing the same pathway.

This study has several limitations. First, we used a cross-sectional design, so a causal relationship cannot be established due to the absence of a temporal context. The association between low HGS and depression may appear large due to re- 
verse causality (muscle weakness due to depressive symptoms). Second, of the 6527 people aged 60 years and older who participated in the KNHANES, only 4274 were selected for this study. However, the excluded participants were more likely to have depression than the included participants (18.1 vs. $26.8 \%$ ), had lower HGS (19.8 vs. 28.2\%), and more prominent HGS asymmetry (12.7 vs. $17.1 \%$ ) (Supplemental Material 4). Thus, the associations that we found might have been even stronger in the excluded participants and our results may therefore be conservative. Even when analyzed with the NOMCAR option of SAS, the results did not differ significantly from the original results (Supplemental Material 5). Nevertheless, since the large amount of missing values might have affected the results, the associations found in this study should be confirmed in additional studies. Lastly, although the PHQ-9 is known to be an effective diagnostic tool for screening of depression, due to the inherent limitations of self-reported measures, differences may exist between the population with depression as defined in this study and the actual population with major depressive disorder.

Despite these limitations, this study also has strengths. Our findings suggest that HGS asymmetry may be related to depression in elderly women. In addition, we found that the HGS asymmetry might strengthen the association between low HGS and depression, and this synergy may be more important than the effect of each factor alone. Lastly, our results suggest that the relationship between HGS and depression is especially prominent in women with certain characteristics, such as being older than 75 years, being normal-weight or underweight, engaging in regular exercise, and having a chronic illness.

Measuring HGS is a cheap, simple, and non-invasive test that can be easily performed in primary care settings. Performing this simple test may help physicians assess both the mental health and physical status of elderly patients. The findings of this study are consistent with previous studies reporting that HGS asymmetry and weakness together are jointly associated with adverse health conditions in older populations [19-21]. Our results suggest that HGS asymmetry has potential as a new indicator of HGS in the elderly, although subsequent studies are needed to confirm its usefulness in clinical settings.

In conclusion, we found that asymmetric or low HGS was associated with depression among Korean elderly women. This association was especially strong when both asymmetric and low HGS were present. Thus, when measuring HGS in the elderly, it is also important to check for symmetry. Although this association should be confirmed in longitudinal studies, our findings support the potential of HGS asymmetry as a new indicator of HGS in the elderly.

\section{SUPPLEMENTAL MATERIALS}

Supplemental materials are available at https://doi.org/10. 3961/jpmph.20.315.

\section{CONFLICT OF INTEREST}

The authors have no conflicts of interest associated with the material presented in this paper.

\section{FUNDING}

None.

\section{ACKNOWLEDGEMENTS}

This study used data from the sixth and seventh KNHNES, 2014, 2016, and 2018, KCDC.

\section{AUTHOR CONTRIBUTIONS}

Conceptualization: KH. Data curation: KH. Formal analysis: KH, YP. Funding acquisition: None. Methodology: KH. YP. Project administration: KH, ECP. Writing - original draft: KH. Writing - review \& editing:YP, GRK, SIJ, ECP.

\section{ORCID}

Kyungduk Hurh https://orcid.org/0000-0002-8295-7767

Yoonsik Park https://orcid.org/0000-0002-8269-0169

Gyu Ri Kim https://orcid.org/0000-0003-3624-3971

Sung-In Jang https://orcid.org/0000-0002-0760-2878

Eun-Cheol Park https://orcid.org/0000-0002-2306-5398

\section{REFERENCES}

1. Korean Statistical Information Service. Population projections for Korea [cited 2020 Jun 5]. Available from: http://kosis.kr/ statHtml/statHtml.do?orgld = 101\&tblld=DT_1BPA003\&co nn_path $=12$ (Korean).

2. Park JH, Kim KW, Kim MH, Kim MD, Kim BJ, Kim SK, et al. A na- 
tionwide survey on the prevalence and risk factors of late life depression in South Korea. J Affect Disord 2012;138(1-2):34-40.

3. Korean Statistical Information Service. Statistical database: health - cause of death (indicator) [cited 2020 Jun 5]. Available from: http://kosis.kr/statisticsList/statisticsListlndex.do? menuld =M_01_01\&vwcd =MT_ZTITLE\&parmTabld = M_ 01_01 (Korean).

4. Organization for Economic Cooperation and Development (OECD). Health at a Glance 2019: OECD indicators [cited 2020 Jun 5] Available from: http://www.oecd.org/health/healthsystems/health-at-a-glance-19991312.htm.

5. Roubenoff R, Hughes VA. Sarcopenia: current concepts. J Gerontol A Biol Sci Med Sci 2000;55(12):M716-M724.

6. Chen LK, Liu LK, Woo J, Assantachai P, Auyeung TW, Bahyah KS, et al. Sarcopenia in Asia: consensus report of the Asian Working Group for Sarcopenia. J Am Med Dir Assoc 2014;15(2):95101.

7. Giampaoli S, Ferrucci L, Cecchi F, Lo Noce C, Poce A, Dima F, et al. Hand-grip strength predicts incident disability in non-disabled older men. Age Ageing 1999;28(3):283-288.

8. Kim GR, Sun J, Han M, Park S, Nam CM. Impact of handgrip strength on cardiovascular, cancer and all-cause mortality in the Korean longitudinal study of ageing. BMJ Open 2019;9(5): e027019.

9. Yang EJ, Lim S, Lim JY, Kim KW, Jang HC, Paik NJ. Association between muscle strength and metabolic syndrome in older Korean men and women: the Korean Longitudinal Study on Health and Aging. Metabolism 2012;61(3):317-324.

10. Kim KH, Park SK, Lee DR, Lee J. The relationship between handgrip strength and cognitive function in elderly Koreans over 8 years: a prospective population-based study using Korean Longitudinal Study of Ageing. Korean J Fam Med 2019;40(1): 9-15.

11. Kim GR, Sun J, Han M, Nam CM, Park S. Evaluation of the directional relationship between handgrip strength and cognitive function: the Korean Longitudinal Study of Ageing. Age Ageing 2019;48(3):426-432.

12. Brooks JM, Titus AJ, Bruce ML, Orzechowski NM, Mackenzie TA, Bartels SJ, et al. Depression and handgrip strength among U.S. adults aged 60 years and older from NHANES 2011-2014. J Nutr Health Aging 2018;22(8):938-943.

13. Han KM, Chang J, Yoon HK, Ko YH, Ham BJ, Kim YK, et al. Relationships between hand-grip strength, socioeconomic status, and depressive symptoms in community-dwelling older adults. J Affect Disord 2019;252:263-270.
14. Crosby CA, Wehbé MA, Mawr B. Hand strength: normative values. J Hand Surg Am 1994;19(4):665-670.

15. Crews Jr WD, Harrison DW, Rhodes RD, Demaree HA. Hand fatigue asymmetry in the motor performances of women with depressed mood. Cogn Behav Neurol 1995;8(4):277-281.

16. Emerson CS, Harrison DW, Everhart DE, Williamson JB. Grip strength asymmetry in depressed boys. Cogn Behav Neurol 2001;14(2):130-134.

17. Merrin EL. Motor and sighting dominance in schizophrenia and affective disorder. evidence for right-hand grip strength prominence in paranoid schizophrenia and bipolar illness. $\mathrm{Br}$ J Psychiatry 1985;146:539-544.

18. Yu J, Rawtaer I, Mahendran R, Kua EH, Feng L. Degree, but not direction of grip strength asymmetries, is related to depression and anxiety in an elderly population. Laterality 2017;22(3): 268-278.

19. McGrath R, Vincent BM, Jurivich DA, Hackney KJ, Tomkinson $G R$, Dahl $L J$, et al. Handgrip strength asymmetry and weakness together are associated with functional disability in aging Americans. J Gerontol A Biol Sci Med Sci 2020:glaa100.

20. Collins K, Johnson N, Klawitter L, Waldera R, Stastny S, Kraemer WJ, et al. Handgrip strength asymmetry and weakness are differentially associated with functional limitations in older Americans. Int J Environ Res Public Health 2020;17(9):3231.

21. McGrath R, Cawthon PM, Cesari M, Al Snih S, Clark BC. Handgrip strength asymmetry and weakness are associated with lower cognitive function: a panel study. J Am Geriatr Soc 2020; 68(9):2051-2058.

22. Korea Centers for Disease Control and Prevention. Korea National Health \& Nutrition Examination Survey: design and methods [cited 2020 Oct 19]. Available from: https://knhanes.cdc. go.kr/knhanes/eng/index.do.

23. Kroenke K, Spitzer RL, Williams JB. The PHQ-9: validity of a brief depression severity measure. J Gen Intern Med 2001;16(9): 606-613.

24. Han C, Jo SA, Kwak JH, Pae CU, Steffens D, Jo I, et al. Validation of the Patient Health Questionnaire-9 Korean version in the elderly population: the Ansan Geriatric study. Compr Psychiatry 2008;49(2):218-223.

25. Innes EV. Handgrip strength testing: a review of the literature. Aust Occup Ther J 1999;46(3):120-140.

26. Lips-Wiersma M, Wright S, Dik B. Meaningful work: differences among blue-, pink-, and white-collar occupations. Career Dev Int 2016;21(5):534-551.

27. World Health Organization. Global Physical Activity Question- 
naire (GPAQ) analysis guide [cited 2020 Oct 5]. Available from: https://www.who.int/ncds/surveillance/steps/resources/GPAQ Analysis_Guide.pdf?ua $=1$.

28. VanderWeele TJ, Knol MJ. A tutorial on interaction. Epidemiol Methods 2014;3(1):33-72.

29. SAS Help Center. SAS/STAT user's guide [cited 2020 Oct 8]. Available from: https://documentation.sas.com/?cdcld=pgm sascdc\&cdcVersion=9.4_3.4\&docsetld = statug\&docsetTarget =statug_surveylogistic_syntax01.htm\&locale=en\#statug. surveylogistic.svlnomcar.

30. Buskirk TD. Estimating design effects for means, proportions and totals from complex sample survey data using SAS ${ }^{\circledR}$ Proc Surveymeans [dissertation]. Saint Louis: Saint Louis University; 2011.

31. Fukumori $N$, Yamamoto $Y$, Takegami M, Yamazaki S, Onishi $Y$, Sekiguchi $M$, et al. Association between hand-grip strength and depressive symptoms: Locomotive Syndrome and Health Outcomes in Aizu Cohort Study (LOHAS). Age Ageing 2015; 44(4):592-598.

32. Schnittker J. Chronic illness and depressive symptoms in late life. Soc Sci Med 2005;60(1):13-23.

33. Hecht D. Depression and the hyperactive right-hemisphere. Neurosci Res 2010;68(2):77-87.
34. Lang PO, Michel JP, Zekry D. Frailty syndrome: a transitional state in a dynamic process. Gerontology 2009;55(5):539-549.

35. Soysal P, Veronese N, Thompson T, Kahl KG, Fernandes BS, Prina $A M$, et al. Relationship between depression and frailty in older adults: a systematic review and meta-analysis. Ageing Res Rev 2017;36:78-87.

36. Vaughan L, Corbin AL, Goveas JS. Depression and frailty in later life: a systematic review. Clin Interv Aging 2015;10:19471958.

37. Portegijs E, Sipilä S, Alen M, Kaprio J, Koskenvuo M, Tiainen K, et al. Leg extension power asymmetry and mobility limitation in healthy older women. Arch Phys Med Rehabil 2005;86(9): 1838-1842.

38. Skelton DA, Kennedy J, Rutherford OM. Explosive power and asymmetry in leg muscle function in frequent fallers and nonfallers aged over 65. Age Ageing 2002;31(2):119-125.

39. Bohannon RW, Magasi SR, Bubela DJ, Wang YC, Gershon RC. Grip and knee extension muscle strength reflect a common construct among adults. Muscle Nerve 2012;46(4):555-558.

40. Aadahl M, Beyer N, Linneberg A, Thuesen BH, Jørgensen T. Grip strength and lower limb extension power in 19-72-year-old Danish men and women: the Health2006 study. BMJ Open 2011;1(2):e000192. 